\title{
High Anion Gap Metabolic Acidosis due to Euglycemic Diabetic Keto- acidosis Caused by Sodium-Glucose Co-transporter 2 inhibitor
}

\author{
Awad Magbri, Eusera El-Magbri, Mariam El-Magbri, Brar Balhinder and Shauket Rashid
}

Toledo Vascular Access Clinic, Toledo, OH, USA

\section{Article Info}

\section{Article Notes}

Received: December 05, 2017

Accepted: December 26, 2017

\section{*Correspondence:}

Dr. Awad Magbri, Toledo Vascular Access Clinic, Toledo, $\mathrm{OH}$ USA; Email: elmagbri@hotmail.com

(c) 2017 Magbri A. This article is distributed under the terms of the Creative Commons Attribution 4.0 International License.

\section{Keywords}

Diabetes mellitus

Diabetic ketoacidosis

Euglycemic diabetic ketoacidosis

Sodium-glucose cotransporter 2 inhibitor

Metabolic acidosis

High anion gap acidosis

\section{Case history}

The case is that of 58 year-male with type 2 diabetes mellitus for 7 years, hypertension, hypercholesterolemia, who was admitted to the hospital with left lower limb cellulitis over the past 8 days. On work-up he was found to have high anion-gap metabolic acidosis (AGMA) with anion gap of 25, his lactate levels were normal (D and L-lactate). He denies overdosing with any medications and his toxicology screen for methanol, ethanol, aspirin, and ethylene glycol were negative. He has no psychiatric history of note. He denies using over the counter medications like acetaminophen. No bowel surgery could be elicited. He felt dehydrated and nauseous but otherwise fine.

His medications includes; carvedalol $25 \mathrm{mg}$ twice daily, hydrochlothiazide $25 \mathrm{mg}$ daily, Lipitor $20 \mathrm{mg}$ daily, insulin, aspirin $81 \mathrm{mg}$ daily, and was started on canagliflozoin $300 \mathrm{mg}$ daily 4 weeks ago to control his blood sugar level and A1C.

Physical examination of the patient revealed, slightly dehydrated but well-nourished man, his vital signs; heart rate of 78 BPM and regular, BP 143/85 mmHg, temperature $98.7 \mathrm{~F}$, and his oxygen saturation while breathing room air was $92 \%$. Examination of the heart, abdomen, and chest were unremarkable. He had left lower leg cellulitis but no edema or tenderness.

His work-up including chemistry-7 which showed sodium of $142 \mathrm{mmol} / \mathrm{L}$, potassium of $4.3 \mathrm{mmol} / \mathrm{L}$, chloride of $102 \mathrm{mmol} / \mathrm{L}$, bicarbonate of $13 \mathrm{mmol} / \mathrm{L}, \mathrm{BUN}$ and creatinine of $18 \mathrm{mg} / \mathrm{L}$ and 0.78 $\mathrm{mg} / \mathrm{L}$ respectively. His blood glucose level was $178 \mathrm{mg} / \mathrm{L}$ with A1C of 8.2. His serum osmolality was $312 \mathrm{mosm} / \mathrm{L}$, and his arterial blood $\mathrm{pH}$ was 7.2 with a carbon dioxide in blood gas analysis (Pco2) of $32 \mathrm{mmHg}$. His calculated anion gap was 25 given his normal albumin level. His investigation also showed positive ketones in the serum and urine. His urine PH was 5.5 and the urine contain $>800 \mathrm{mg}$ of glucose.

\section{Case discussion}

Metabolic acidosis is defined as low $\mathrm{pH}<7.3$ with serum bicarbonate level of $<22 \mathrm{mmol} / \mathrm{L}$. Diabetic ketoacidosis (DKA) is one of the serious and acute complications of diabetes. It is characterized by increased total body ketone levels, metabolic acidosis, and hyperglycemia. Hyperglycemia is a key component for the diagnosis 
of DKA. There is another DKA with normal blood glucose described first by Munro in 1973; where he reported 37 episodes of DKA with normal or near normal blood glucose in 17 young patients ${ }^{1}$. Later on normoglycemia is defined as blood sugar $<250 \mathrm{mg} / \mathrm{L}$. It is now known as Euglycemic diabetic ketoacidosis (eDKA). Burge et $\mathrm{al}^{2}$ have demonstrated that fasting in type 1 diabetic patients can lead to blunted hyperglycemic response which can lead to eDKA. These researchers demonstrated that dehydration can enhance the development of eDKA ${ }^{3}$.

Euglycemic DKA is part of the spectrum of DKA and can be distinguished from other causes of ketoacidosis by clinical history and serum bicarbonate levels. The level of bicarbonate in starvation ketosis is usually normally more than $18 \mathrm{mEq} / \mathrm{L}$. The absence of other causes of high anion gap metabolic acidosis like lactic acidosis, drug intoxications, and renal failure as well as the presence of ketones in the plasma and urine distinguish eDKA from other causes of $\mathrm{AGMA}^{4}$. Many workers demonstrated that eDKA can occur in both type 1 and type 2 diabetes Mellitus $^{5}$. The most common causes that precipitated DKA are infections $s^{6-8}$, inadequate insulin therapy, pancreatitis, myocardial infarction, and illicit drug use $\mathrm{e}^{8-10}$. The American Diabetic Association, defined DKA as plasma glucose of $>250 \mathrm{mg} / \mathrm{L}$, positive urinary or serum ketones, arterial $\mathrm{PH}$ of $<7.3$, serum bicarbonate $<18 \mathrm{mEq} / \mathrm{L}$, and high anion gap.

The case under discussion satisfy all the criteria of DKA except hyperglycemia. In 2013, the food and drug administration (FDA) approved many sodium-glucose cotransporter 2 inhibitor (SGLT-2 inhibitors) for management of type -2 diabetes mellitus. The use of these drugs in type-1 and 2-DM have increased the side effects and the risks of eDKA in these patients ${ }^{11-13}$. The adverse effects of these drugs were recorded in 73 cases linked to eDKA. All patients required hospitalization and their average blood glucose level was $211 \mathrm{mg} / \mathrm{L} .60 \%$ of these patients have diabetes type- 2 . SGLT- 2 inhibitors in these patients were started on average of 45 days prior to the incident. The majority of the cases had infection, dehydration, and de-escalation of insulin doses. The management of these patients were in the same line as patients with classic DKA with rehydration, insulin supplementation and treatment of the underlying precipitating cause. In some of these patients re-challenged with SGLT-2 inhibitors resulted in the development of eDKA ${ }^{11}$.

Restriction of carbohydrate usage because of infection or dehydration along with de-escalation of the insulin doses because of normal blood sugars, and excretion of large amount of blood glucose in the urine as part of the effect of SGLT- 2 inhibitors are suggested as the basis of the pathogenesis of eDKA. These factors increased reliance on the fat oxidation for energy with ensuing ketoacidosis ${ }^{14,15}$.
Treatment of DKA and eDKA are the same and includes;

1. volume resuscitation, frequent assessment of cardiac function, renal function, and mental status and avoidance of fluid overload and pulmonary edema $^{16}$

2. Low dose intra-venous regular insulin.

3. Correction of potassium abnormality

4. Resolution of DKA can be assessed by the presence of 2 of the following: an anion gap $<12$, serum bicarbonate level $>15$, or a venous $\mathrm{pH}>7.3^{4,17}$

The important features in eDKA is the perception by the patient and the health care individual that normal blood sugar resulted in the de-escalation of the insulin dose and perpetuation of the eDKA.

Awareness that DKA can occur without high blood sugars is critical and blood gas analysis for AGMA as well as estimation of serum and urine ketones are keys for the correct diagnosis of eDKA and hence, successful management of the condition.

In conclusion: all patients with type- 1 or 2 diabetes on SGLT-2 inhibitors with nausea, vomiting, shortness of breath, or malaise should have chemistry-7, blood gas analysis, and urine and serum ketones estimation to avert the complications of eDKA.

\section{References}

1. Munro JF, Campbell IW, McCuish AC, et al. Euglycemic diabetic ketoacidosis. BMJ. 1973; 2: 578-80.

2. Burge MR, Hardy KJ, Schade DS. Short-term fasting is a mechanism for the development of Euglycemic ketoacidosis during periods of insulin deficiency. J Clin Endocrinol Metab. 1993; 76: 1192-98.

3. Burge MR, Garcia N, Quall CR, et al. Deferential effects of fasting and dehydration in the pathogenesis of diabetic ketoacidosis. Metabolism. 2001; 50: 171-77.

4. Kitabchi AE, Umpierrez GE, Miles JM, et al. Hyperglycemic crises in adult patients with diabetes. Diabetic Care. 2009; 32: 1335-43.

5. Rawla P, Vellipuram AP, Bandaru SS, et al. Euglycemic diabetic ketoacidosis: a diagnostic and therapeutic dilemma. Endo. Diab \& Metabol. 2017; 1-4. http://www.edmcasereports.com

6. National center for health statistics. National hospital discharge and ambulatory surgery data (article online) available from http://www. cdc.gov/nchs/about/major/hdasd/nhds.htm.

7. Kitabchi AE, Umpierrez GE, Murphy MB, et al. Management of hyperglycemic crises in patients with diabetes. Diabetic care. 2001; 24: $131-153$

8. Ennis ED, Stahl EJVB, Kreisberg RA. The hyperosmolar hyperglycemic syndrome. Diabetes Rev. 1994; 2: 115-26.

9. Kitabchi AE, Umpierrez GE, Murphy MB, et al. Hyperglycemic crises in adult patients with diabetes. Diabetes Care. 2006; 29: 2739-2748

10. Defronzo RA, Matzuda M, Barret E. Diabetic ketoacidosis: a combined metabolic-nephrologic approach to therapy. Diabetes Rev. 1994; 2: 209-238 
11. Peters AL, Buschur EO, Buse JB, et al. Euglycemic diabetic ketoacidosis: A potential complication of treatment with sodium-glucose cotransporter 2 inhibition. Diabetic Care. 2015; 38(9): 1687-93.

12. Henry, Robert R, et al. "Efficacy and safety of the canagliflozoin, a sodium-glucose co-transporter 2 inhibitor, as add-on to insulin in patients with type 1 diabetes." Diabetes care. 2015; 38(12): 2258-2267.

13. "Euglycemic diabetic ketoacidosis: a predictable, detectable, and preventable safety concern with SGLT 2 inhibitor." Diabetes Care. 2015(39.9): 1638-42. US Food and Drug Administration. "FDA Drug Safety Communication: FDA warns that SGLT 2 inhibitors for diabetes may result in a serious condition of too much acid in the blood." Bethesda. MD: Food and Drug Administration. 2015 May 15 (2015).
14. Hayami T, Kato Y, Kamiya H, et al. Case of ketoacidosis by a sodiumglucose cotransporter 2 inhibitor in a diabetic patient with low carbohydrate diet. J Diabetes Invest. 2015; 10: 12330

15. St Hilaire R, Costello H. Prescriber beware: report of adverse effects of sodium-glucose cotransporter 2 inhibitor use in a patient with contraindication. Am J Emerg Med 2015; 33: 604. E3-e4.

16. Savage MW, Dhatariya KK, Kilvert A, et al. Joint British Diabetes Societies. Joint British Diabetes Societies guideline for the management of diabetic ketoacidosis. Diabet Med. 2011; 28: 508-15.

17. Jenkins D, Close CF, Krentz AJ, et al. Euglycemic diabetic ketoacidosis: Does it exist. Acta Diabetol. 1993; 30: 251-53. 